\title{
Ringfalten an der Stutenzitze
}

\author{
Jutta Friker, J. P. Ehlers', Eva Zeiler² und H.-G. Liebich
}

Institut für Tieranatomie, Ludwig-Maximilians-Universität, München, Gynäkologische und Ambulatorische Tierklinik, Ludwig-Maximilians-Universität, München und Institut für Nutztierwissenschaften, Universität für Bodenkultur, Wien²

\section{Zusammenfassung}

An 15 Eutern frisch geschlachteter, klinisch unauffälliger Stuten wurde untersucht, ob morphologische Sonderbildungen zur Infektionsabwehr ausgebildet sind. Hierzu wurden neben Gefrierschnitten, Korrosionspräparaten und histologischen Schnittpräparaten auch Abstriche aus dem Zitzenkanal angefertigt und beurteilt. An der Stutenzitze befindet sich proximal der Grenze zwischen Pars papillaris sinus lactiferi und Ductus papillaris eine Ringfalte, die in unterschiedlicher Stärke ausgebildet ist. In den Tupferproben der Abstriche aus der Stutenzitze wurde eine Proliferation und Keratinisierung des Epithels nachgewiesen. Als Hypothesen für die Funktion der Ringfalten werden eine Verbesserung der Abwehrlage sowie eine Beteiligung am Saugakt angeführt. Die Schollen in den Abstrichen werden als Indiz für den Ablauf von infektionsabwehrenden Hyperkeratoseprozessen gewertet. Die vorliegende Arbeit soll als Beitrag zur Ergänzung des Wissens um das bisher recht spärlich untersuchte Stuteneuter dienen.

Schlüsselwörter: Stute, Zitze, Ringfalte, Hyperkeratose, Anatomie

\section{Circular folds in the mare's teat}

15 non pathological udders of freshly slaughtered mares were taken to investigate whether there are morphological structures on behalf of an infection defence in mare's teat. Therefore frozen sections, corrosion-cast and slight preparations were made. Furthermore smears of the Pars papillaris sinus lactiferi were taken for cytological examination. In mare's teat proximal the border between the Pars papillaris sinus lactiferi and the Ductus papillaris a ring-formed fold is developed. In the swab samples proliferation and keratinisation of cells was found. As a hypothesis the function of the ring-formed folds could be to increase the infection defence in the udder and respectively or they could take part in the act of sucking. The results of the swab samples are rated as a sign of an active process of resistance to infection. This article is a contribution to advance the knowledge of the morphological structures in mare's teat.

Keywords: mare, teat, circular fold, hyperkeratosis, anatomy

\section{Einleitung}

Die Beschaffenheit des Präkolostrums lässt bei Stuten einen Rückschluss auf den nahenden Geburtstermin zu (Karbaum 2000). Befürchtungen, wonach die für die Sekretgewinnung notwendigen Manipulationen am Stuteneuter zu Mastitiden führen könnten, erwiesen sich dabei als unbegründet.

In der Literatur finden sich keine Angaben über Abwehrmechanismen gegen Infektionserreger am Stuteneuter im Allgemeinen und der Stutenzitze im Speziellen (Lau 2000). Es wurde deshalb untersucht, ob und wenn ja welche morphologischen Strukturen an der Stutenzitze ausgebildet sind, die der Abwehr von Keimen dienen. Insbesondere wurde dabei, in Anlehnung an die Kenntnisse vom gut untersuchten Rindereuter, auf Einrichtungen, wie der Fürstenberg'schen Rosette, dem Fürstenberg'schen Venenring oder diesen vergleichbaren Strukturen, geachtet. Eine zytologische Analyse sollte zusätzlich Aufschluss über das Vorliegen von Hyperkeratosevorgängen geben, deren primäre Aufgabe die Infektabwehr ist.

\section{Material und Methode}

Es wurden 15 Euter untersucht, die von geschlachteten, klinisch unauffälligen Stuten (Alter: 5 - 24 Jahre, 13 Tiere über
9 Jahre; Rasse: 11 Warmblutstuten, je eine Haflinger-, Traber-, Araber- und Islandponystute) stammten.

Von jedem Euter wurde, unmittelbar nach der Schlachtung der Stuten, eine der beiden Zitzen präpariert und ein Abstrich aus dem Zitzenkanal entnommen. Hierfür wurde ein in physiologischer Kochsalzlösung getränkter Wattetupfer verwendet. Diese Ausstriche der Tupferproben wurden nach Papanicolaou gefärbt und die vorhandenen Zellen lichtmikroskopisch bestimmt.

In die Zitzen der zweiten Euterhälfte, wurde bei zehn ein Venenverweilkatheter (Vasodrop ${ }^{\circledR}$, Querdurchmesser 1,7 mm, Länge $45 \mathrm{~mm}$ ) eingeführt und fünf von diesen mit Gelatinelösung (10 Teile Gelatine + 90 Teile Wasser + 0,5 Teile Farbstoff) sowie fünf mit gefärbtem Rekli Injektionsharz gefüllt. Die Gelatinepräparate wurden in gefrorenem Zustand (Tiefkühlung auf $-20^{\circ}$ C) mit einer Bandsäge geschnitten und zunächst mit Gelatinefüllung und darauf, nach Auswaschen der Gelatine, ohne Füllung photographiert. Von den Harzpräparaten wurde, nach Aushärtung des Injektionsharzes, das oberflächliche Gewebe der Milchdrüsen abpräpariert und die Organe anschließend im Wasserbad bei $+70^{\circ} \mathrm{C}$ mazeriert. Die restlichen 5 Zitzen wurden vom Drüsenkörper abgetrennt und zur Herstellung von histologischen Schnittpräparaten 
zunächst in Bouin fixiert. Zur Beurteilung der Gewebe wurden Färbungen nach Goldner (Trichromfärbung) und H.E. verwendet.

\section{Ergebnisse}

Anhand der Makrogefrierschnitte (Abb. 1 und 2) und Korrosionspräparate (Abb. 3) war bei allen zehn Stutenzitzen eine Ringfaltenbildung im distalen Drittel der Pars papillaris sinus lactiferi, wenn auch in unterschiedlicher Ausprägung, nachzuweisen.

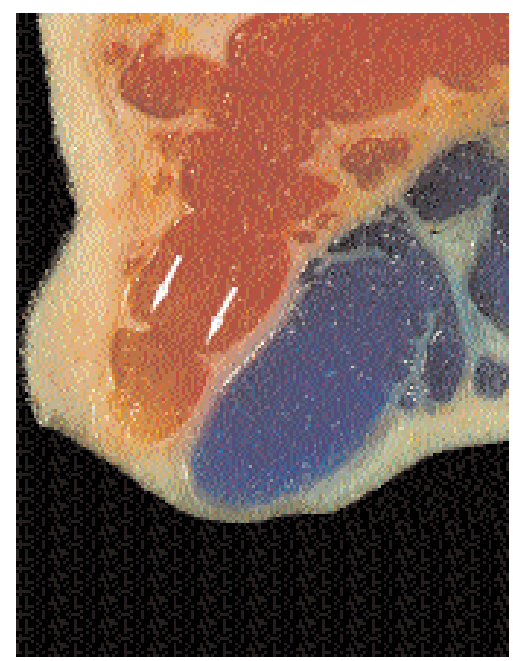

Abb 1 Gefrierschnitt eines Gelatineausgusses. Die Pfeile zeigen auf die Ringfalte.

Frozen section of a teat, filled with gelatine. The arrows mark the ring-formed fold.

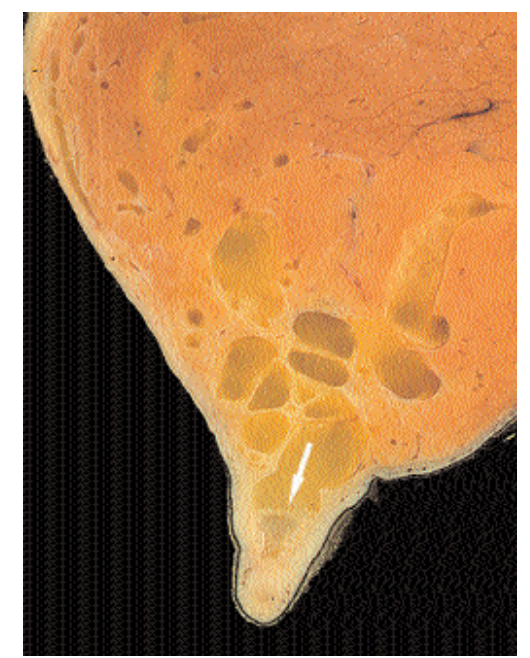

Abb 2 Gefrierschnitt eines Gelatineausgusses, nach Entfernen der Gelatine. Der Pfeil zeigt auf die Ringfalte.

Frozen section of a teat. The arrow marks the ring-formed fold.

Die Resultate der histologischen Untersuchung bestätigten den makroskopischen Befund. Es handelte sich um faltenförmige Vorwölbungen, bestehend aus der oberflächlichen Schleimhaut und der darunter liegenden Gewebsschicht (Abb. 4).

In der zytologischen Untersuchung zeigten sich Epithelzellen in unterschiedlichen Stadien des Zelluntergangs. Es konnten Inter- mediär- und Superfizialzellen sowie keratinisierte Schollen beobachtet werden (Abb. 5). Ein Fürstenberg'scher Venenring oder eine ähnliche Struktur konnte nicht festgestellt werden. Als Nebenbefund ist herauszustellen, dass drei der 15 untersuchten Euter jeweils auf beiden Zitzen nur ein Ostium papillare aufwiesen. Eine Nachpräparation zeigte, dass in diesen Fällen auch nur ein Drüsenkomplex pro Euterhälfte angelegt war.

\section{Diskussion}

In der vorliegenden Untersuchung konnte die Ausbildung von Ringfalten an der inneren Oberfläche der Stutenzitze nachge-

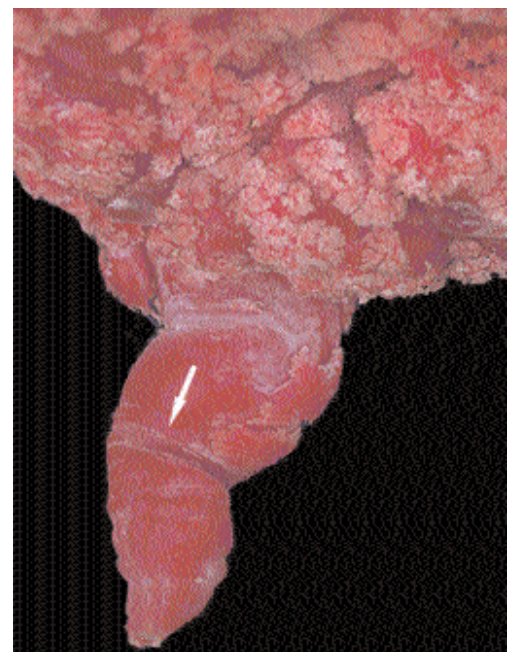

Abb 3 Korrosionspräparat einer Stutenzitze. Der Pfeil zeigt auf die Ringfalte.

Corrosion-cast preparation of a teat. The arrow marks the ringformed fold.

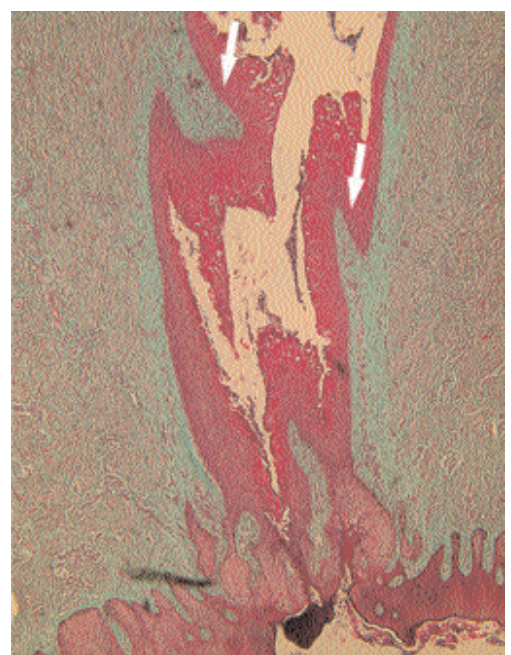

Abb 4 Längsschnitt durch eine Zitze. Färbung: Die Pfeile zeigen auf die Ringfalte. Färbung: Trichrom nach Goldner. Longitudinal-section of a teat. (Staining method: Trichrome of Goldner). The arrows mark the ring-formed folds.

wiesen werden. Funktionell liegt die Vermutung nahe, dass diese Gebilde dem Abwehrsystem der Zitze zuzuordnen sind. Die Ringfalten befinden sich proximal der inneren Öffnung des Ductus papillaris. Es bleibt fraglich, ob sie als Analogon zur Fürstenberg'schen Rosette des Rindes angesehen werden dürfen, da diese an der Grenze der Pars papillaris sinus lactiferi zum Ductus papillaris lokalisiert ist. Die Falten des Stu- 
teneuters bedingen aber auf jeden Fall eine Passageverlängerung in der Stutenzitze und können somit als ein mechanisches Hindernis für eindringende Bakterien und Keime angesehen werden.

Auch der Nachweis der Proliferation des Epithels mit der Bildung von keratinisierten Schollen ist unter dem Gesichtspunkt einer Verbesserung der Abwehrlage am Stuteneuter zu sehen. Durch die Proliferation kommt es zur Abschilferung von Zellen und damit zur Ausschwemmung von eindringenden oder anhaftenden Erregern. Die Keratinisierung erschwert das Eindringen von Keimen ins Epithel.

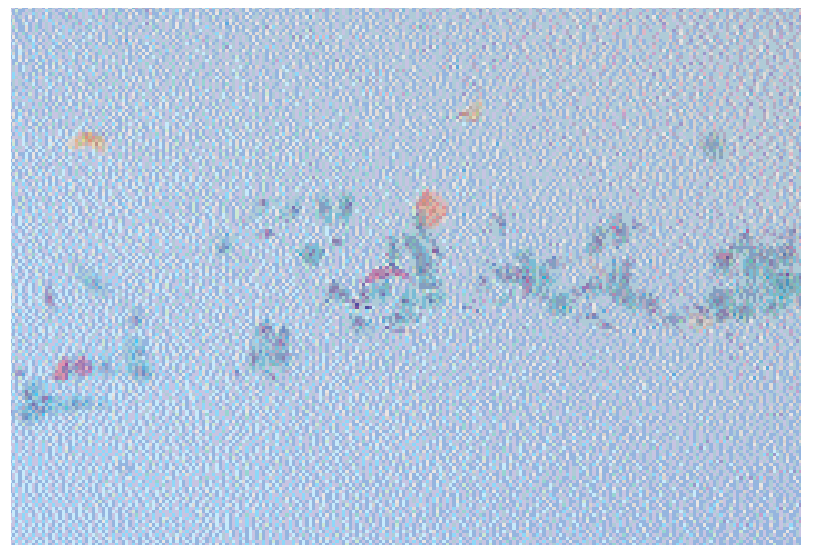

Abb 5 Zellen eines Zitzenkanalabstrichs einer Stute. Färbung Papanicolaou.

Cells of the Pars papillaris sinus lactiferi in mares. (Staining method: Papanicolaou).

Die Kenntnis über Abwehrmechanismen an der Milchdrüse der Stute ist für den Kliniker bedeutsam. Für ihn spielt sie für diagnostische Zwecke, für die Bestimmung des nahenden Geburtstermins, für die Bereitstellung von Kolostralmilch für ein Neugeborenes, das vom Muttertier nicht angenommen wurde sowie bei der Gewinnung von Milch für Allergiker eine Rolle. Die hier vorgestellten Ergebnisse werden deshalb in weiterführenden Untersuchungen präzisiert und um die Abwehrmechanismen am gesamten Stuteneuter ergänzł werden.

Als alternative oder zusätzliche Funktion der Ringfalten ist eine Beteiligung am Saugakt denkbar. Da hier, anders als beim Kalb, weniger Unterdruck aufgebaut wird (Scheunert und Trautmann 1987; Cameron et al. 1999), könnten die Falten an der Regulation der Milchabgabemenge beteiligt sein.
Der in der Untersuchung erhobene Nebenbefund, dass bei drei der 15 untersuchten Euter nur ein Ostium papillare pro Euterhälfte mit nur einem Drüsenkomplex ausgebildet war, ist unter klinischen Aspekten beachtenswert. In derart gelagerten Fällen ist die lokale Applikation von Therapeutika vereinfacht. Die Möglichkeit der Ausbildung von nur einem Ostium papillare ist in den einschlägigen Abschnitten der gängigen deutschsprachigen Lehrbücher der Tieranatomie (Habermehl 1996, Dyce et al. 1997, Budras und Röck 2000, Bragulla et al. 2002, Bartmann et al. 2002) nicht erwähnt. Hier wird die Anzahl der ausgebildeten Ostia papillaria mit 2 bis 3 beziffert, eine Angabe, die für ca. $80 \%$ aller Stuten zuzutreffen scheint.

\section{Literatur}

Bartmann C. P., H. Wissdorf und K. Übermuth (2002): Weibliche Geschlechtsorgane, Milchdrüse und Harnröhre. In: Wissdorf H., H. Gerhards, B. Huskamp und E. Deegen (2002): Praxisorientierte Anatomie und Propädeutik des Pferdes, 2. Aufl., Schaper Verlag, Hannover

Bragulla H., K.-D. Budras, C. Müllig, S. Reese und H. E. König (2002): Allgemeine Körperdecke. In: König H. E. und H.-G. Liebich (2002): Anatomie der Haussäugetiere, 2. Aufl., Schattauer Verlag, Stuttgart, New York

Budras K.-D. und S. Röck (2000): Atlas der Anatomie des Pferdes Lehrbuch für Tierärzte und Studierende, 4. Aufl., Schlüterscher Verlag, Hannover

Cameron E. Z., K. J. Stafford, W. L. Linklater und C. J. Veltman (1999): Suckling behaviour does not measure milk intake in horses, Equus caballus. Animal Behavior, 57 (3), 674 - 678

Dyce M., W. O. Sack und C. J. G. Wensing (1997): Anatomie der Haustiere, Enke Verlag, Stuttgart

Habermehl K.-H. (1996): Haut und Hautorgane in: Nickel R., A. Schummer und E. Seiferle (1996): Lehrbuch der Anatomie der Haustiere, Bd. III, 3. Aufl., Parey Verlag, Berlin, Hamburg

Karbaum E. (2000): Präkolostrum und Geburt bei der Stute, Vet. Med. Diss. Universität München

Lau P. (2000): Zur Anatomie der weiblichen Milchdrüse bei den Haussäugetieren, Vet. med. Diss. Universität München

Scheunert A. und A. Trautmann (1987): Lehrbuch der VeterinärPhysiologie, 7. Aufl., Paul Parey Verlag, Berlin, Hamburg

\title{
Pferdeheilkunde Forum 2004 - Berliner Fortbildungstage
}

\author{
Die BWL-Trilogie
}

\author{
"Finanzmanagement und Controlling, Mitarbeiterführung und Kommunikation \\ und grundlegende Erfolgsstrategien für die tierärztliche Praxis"
}

3 Blockseminare am Donnestag 10. Juni 2004, 11-14 Uhr, 15-18 Uhr und 18.30 bis 21 Uhr 